Original Research Paper

\title{
A Complex Multi-State System with Vacations in the Repair
}

\author{
Juan Eloy Ruiz-Castro \\ Department of Statistics and Operational Research and IeMath Granada, Faculty of Science, \\ University of Granada, Campus Fuentenueva s/n. 18071, Spain
}

Article history
Received: 14-07-2019
Revised: 21-08-2019
Accepted: 04-09-2019

Email: jeloy@ugr.es

\begin{abstract}
A complex multi-state system subject to wear failure and given preventive maintenance is considered. Various internal levels of degradation are assumed. The repair facility is composed of a repairperson, who may take one or more vacations during the period considered. A policy is established for the repairperson's vacation time. Two types of task may be performed by the repairperson: corrective repair and preventive maintenance. All embedded times in the system are phase type distributed. The transient and stationary distributions are determined and several reliability measures are developed in a matrix-algorithmic form. Costs and rewards are included in the model. The results are implemented computationally with Matlab. A numerical example shows that the distribution of vacation time can be optimised according to the net reward established.
\end{abstract}

Keywords: Phase-Type Distribution, Preventive Maintenance, Optimal Vacation Time

\section{Introduction}

In reliability literature, the system in which a unit performs is usually considered in terms of traditional binary models: the up state (performing) vs. the down state (failure). However, many real life systems are composed of different performance levels and incorporate multiple events. To reflect this reality, binary systems have been extended to create multi-state systems, a concept introduced by Murchland (1975). These systems are of particular importance in ensuring reliability and have a finite number of performance/degradation stages. Several methods have been considered to study this approach, including Markov and semi-Markov models, generating functions, Lztransform and Monte Carlo simulations (Lisnianski et al., 2018). Lisnianski and Frenkel (2012) included Markov processes in an analysis of multi-state systems, highlighting the benefits of their application and RuizCastro and Dawabsha (2018) recently modelled a complex multi-state system subject to multiple events, namely Markovian Arrival Processes.

In order to improve system reliability and to increase profits, preventive maintenance is often incorporated into a multi-state system. Nakagawa (1977) reviewed and summarised his own initial studies in this regard and later examined standard and advanced maintenance policies for system reliability in Nakagawa (2005). Preventive maintenance has also been included in complex systems, such as the cold standby two-component system based on semi-Markovian processes that was discussed by Zhong and Jin (2014). In other approaches, complex redundant systems maintained by one or more repairpersons have been considered (Ruiz-Castro and Dawabsha (2018; RuizCastro, 2018; 2016a; 2016b)).

Intractable expressions of difficult interpretation may appear when complex systems are modelled, but they can be analysed in a well structured way, with a matrixalgebraic form, using a phase-type distribution. These distributions were first proposed by Neuts $(1975 ; 1981)$, who highlighted their useful algorithmic properties. Phase type distributions and Markov processes have been applied in fields such as queuing theory, survival and reliability, where real-life problems have been modelled in an algorithmic form by Ruiz-Castro and Fernández-Villodre (2012).

Sometimes, when repairable reliability systems are considered, the fact of limited maintenance resources may conflict with the need for maintenance. To address this situation, Takagi (1991) introduced the theory of a repairperson taking multiple vacations. Under this theory, a repairperson can take a vacation and enter the next vacation immediately if the system functions normally, or under different situations. The term vacation means that sometimes the system will not be repaired immediately after it fails. In this respect, Arulmozhi and Nadarajan (1994) (among others) studied a parallel system with multiple repairpersons taking multiple vacations. Vacations models are given in Tian and Zhang (2006).

In the present paper, we model a discrete multi-state system subject to wear failure and receiving preventive maintenance from a repairperson taking multiple 
vacations. Random vacation time is introduced in this paper to maximize the profit of a system. Two different tasks can be carried out by the repairperson: corrective repair and preventive maintenance. All embedded time distributions are phase type distributed. Costs and rewards are introduced and various measures are obtained, both in a transient and in a stationary regime. The vacation time distribution is optimised according to net reward and a numerical example is given.

The rest of this paper is organised as follows. Section 2 outlines the system, after which it is modelled in detail using a vector Markov process in Section 3. In Section 4, the transient and stationary distributions are calculated by matrix-analytics methods. Section 5 is focused on calculating the reliability measures in transient and stationary regimes. Cost and rewards are defined in Section 6 and then, in Section 7, two numerical examples are provided to show the versatility of the model, in which the vacation time distribution is optimised. Finally, the main conclusions drawn are summarised in Section 8.

\section{The System}

We assume a multi-state system with multiple phases which are partitioned into three operational stages according to the damage sustained: minor, moderate or major damage. This system is subject to repairable failures and undergoes preventive maintenance. The repair facility is composed of a single repairperson who at any time may be observing the system or may be on vacation. The following policy is adopted with respect to vacations: initially, when the system is new, the repairperson is on vacation. Subsequently, any one of the four following cases may occur when the repairpeson arrives:

1. The system suffers minor damage and the repairperson begins vacations again.

2. The system suffers moderate damage and then the repairperson remains in place, waiting to see if a transition to major damage occurs or if the system fails. In the former case, preventive maintenance begins and if a failure occurs, the repairperson begins the corrective repair.

3. The system suffers major damage and preventive maintenance begins.

4. The system fails and corrective repair begins.

After repairing the repairperson goes on vacations.

We assume that the embedded times in the system are phase type distributed. Thus, the operational time has the representation $(\boldsymbol{\alpha}, \mathbf{T})$ with $n$ phases, the vacations time distribution $(v, \mathbf{V})$, with $w$ phases, the corrective repair time $\left(\boldsymbol{\beta}^{1}, \mathbf{S}_{1}\right)$, with order $z_{1}$ and the preventive maintenance time distribution $\left(\boldsymbol{\beta}^{2}, \mathbf{S}_{2}\right)$, with order $z_{2}$.

The number of phases for each damage level is $n_{1}, n_{2}$ and $n_{3}$ for minor, middle and major damage respectively.
The operational time is partitioned into three damage levels and the corresponding matrix $\mathbf{T}$ and initial distribution $\alpha$ is partitioned as follows:

$$
\boldsymbol{T}=\left(\begin{array}{lll}
\boldsymbol{T}_{11} & \boldsymbol{T}_{12} & \boldsymbol{T}_{13} \\
\boldsymbol{T}_{21} & \boldsymbol{T}_{22} & \boldsymbol{T}_{23} \\
\boldsymbol{T}_{31} & \boldsymbol{T}_{32} & \boldsymbol{T}_{33}
\end{array}\right) ; \quad \boldsymbol{T}^{0}=\left(\begin{array}{l}
\boldsymbol{T}_{1}^{0} \\
\boldsymbol{T}_{2}^{0} \\
\boldsymbol{T}_{3}^{0}
\end{array}\right)
$$

Given a matrix $\mathbf{A}$, throughout this paper $\mathbf{A}^{0}$ will denote the column vector $\mathbf{e}-\mathbf{A e}$, where $\mathbf{e}$ is a column vector of ones with appropriate order.

\section{The Modeling}

The system is governed by a vector Markov process with a state-space composed of the following macro-states:

$$
S=\left\{\begin{array}{l}
1=O_{1}, 2=O_{2}^{W R}, 3=O_{2}^{R}, 4=O_{3}^{W R}, \\
5=P M, 6=F^{W R}, 7=C R
\end{array}\right\}
$$

The macro-states denote the following situation:

$O_{1}=$ The system is working at a minor damage level

$O_{2}^{W R}=$ The system is working at a moderate damage level and the repairperson is on vacation

$O_{2}^{R}=$ The system is working at a moderate damage level and the repairperson is in the workspace

$O_{3}^{W R}=$ The system is working at a major damage level and the repairperson is on vacation

$P M=$ The repairperson is engaged in preventive maintenance

$F^{W R}=$ The system is broken and the repairperson is on vacation

$C R=$ The repairperson in engaged in corrective repair

These macro-states are partitioned into operational states:

$$
W=\left\{1=O_{1}, 2=O_{2}^{W R}, 3=O_{2}^{R}, 4=O_{3}^{W R}\right\},
$$

and failure states:

$$
F=\left\{5=P M, 6=F^{W R}, 7=C R\right\} .
$$

Therefore, $S=W \cup \mathrm{F}$.

The transition probability matrix is a block-matrix whose structure is described as follows:

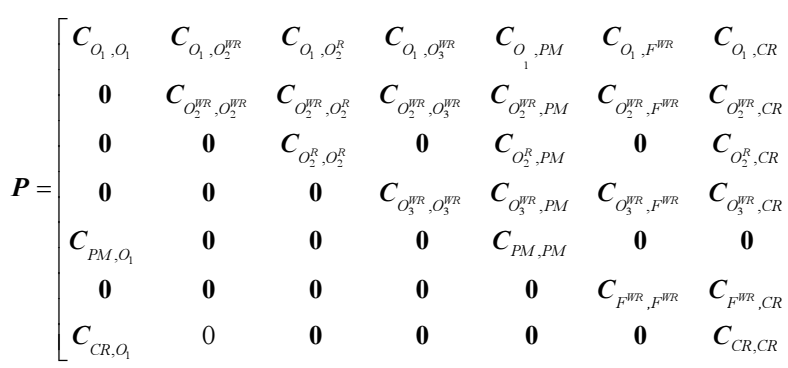


Here, we proceed in explaining in detail three of the matrix blocks as regards the transitions $O_{1} \rightarrow O_{1}, O_{3}^{W R} \rightarrow P M$ and $C R \rightarrow O_{1}$ and then the rest of the matrix blocks are briefly presented.

The transition $O_{l} \rightarrow O_{l}$ is governed by the matrix $\boldsymbol{C}_{\boldsymbol{O} l, \boldsymbol{O}}$. This matrix contains the phases from the macrostate to that when the system is operational after undergoing minor damage $\left(\mathbf{T}_{11}\right)$ and then returns to the macro-state. This situation arises when the system continues working after minor damage occurs and the repairperson remains on vacation $(\mathbf{V})$ or otherwise returns and then begins another vacation $\left(\mathbf{V}^{0} v\right)$. Therefore:

$$
\boldsymbol{C}_{O_{1}, O_{1}}=\boldsymbol{T}_{11} \otimes\left(\boldsymbol{V}+\boldsymbol{V}^{0} \mathrm{v}\right)
$$

In the transition $O_{3}^{W R} \rightarrow P M$, the system initially continues working after undergoing major damage and the repairperson is on vacation. In the next step, the system does not fail $\left(\boldsymbol{e}-\boldsymbol{T}_{3}^{0}\right)$ and the repairperson's vacation ends $\left(\mathbf{V}^{0}\right)$. The repairperson observes that the system has undergone major damage and begins the preventive maintenance $\left(\boldsymbol{\beta}^{2}\right)$. Therefore:

$$
\boldsymbol{C}_{O_{3}^{\boxplus R,}, P M}=\left(\boldsymbol{e}-\boldsymbol{T}_{3}^{0}\right) \otimes V^{0} \otimes \beta^{2} .
$$

In the transition $C R \rightarrow O_{1}$, the system is receiving corrective repair. In the next step, the repair concludes $\left(S_{1}^{0}\right)$, the system begins working $\left(\alpha_{1}\right)$ and the repairperson begins a new vacation $(v)$. Therefore, the matrix block is given by:

$$
\boldsymbol{C}_{C R, O_{1}}=\boldsymbol{\alpha}_{1} \otimes \boldsymbol{S}_{1}^{0} \otimes \mathbf{v}
$$

The rest of the matrix blocks are given by the following expressions:

$$
\begin{aligned}
& C_{O_{1}, O_{2}^{\text {WR }}}=\boldsymbol{T}_{12} \otimes \boldsymbol{V} \quad ; \quad \boldsymbol{C}_{O_{1}, O_{2}^{\mathrm{p}}}=\boldsymbol{T}_{12} \otimes \boldsymbol{V}^{0} \\
& \boldsymbol{C}_{O_{1} O_{3}^{\mathbb{T} R}}=\boldsymbol{T}_{13} \otimes \boldsymbol{V} \quad ; \quad \boldsymbol{C}_{O_{1}, P M}=\boldsymbol{T}_{13} \boldsymbol{e} \otimes \boldsymbol{V}^{0} \otimes \beta^{2} \\
& \boldsymbol{C}_{O_{1}, F^{F R}}=\boldsymbol{T}_{1}^{0} \otimes \boldsymbol{V} \quad ; \quad \boldsymbol{C}_{O_{1}, C R}=\boldsymbol{T}_{1}^{0} \otimes \boldsymbol{V}^{0} \otimes \beta^{1} \\
& \boldsymbol{C}_{O_{2}^{\boxplus R R}, O_{2}^{\Pi R}}=\boldsymbol{T}_{22} \otimes \boldsymbol{V} \quad ; \quad \boldsymbol{C}_{O_{2}^{\text {TrR }}, O_{2}^{R}}=\boldsymbol{T}_{22} \otimes \boldsymbol{V}^{0}
\end{aligned}
$$

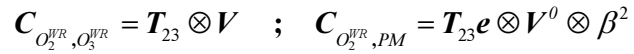

$$
\begin{aligned}
& \boldsymbol{C}_{O_{2}^{\boxplus R R}, F^{W R}}=\boldsymbol{T}_{2}^{0} \otimes \boldsymbol{V} \quad ; \quad \boldsymbol{C}_{O_{2}^{\text {IR }}, C R}=\boldsymbol{T}_{2}^{0} \otimes \boldsymbol{V}^{0} \otimes \beta^{1} \\
& \boldsymbol{C}_{O_{2}^{R}, O_{2}^{R}}=\boldsymbol{T}_{22} \quad ; \quad \boldsymbol{C}_{O_{2}^{R}, P M}=\boldsymbol{T}_{23} \boldsymbol{e} \otimes \beta^{2} \\
& \boldsymbol{C}_{O_{2}^{R}, C R}=\boldsymbol{T}_{2}^{0} \otimes \beta^{1} \quad ; \quad \boldsymbol{C}_{O_{3}^{\text {WR }}, O_{3}^{\text {WR }}}=\boldsymbol{T}_{33} \otimes \boldsymbol{V}
\end{aligned}
$$

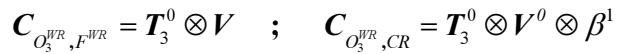

$$
\begin{aligned}
& \boldsymbol{C}_{P M, O_{1}}=\alpha_{1} \otimes S_{2}^{0} U ; \quad \boldsymbol{C}_{P M, P M}=\boldsymbol{S}_{2} \\
& \boldsymbol{C}_{F^{H R}, F^{W^{N R}}}=\boldsymbol{V} \quad ; \quad \boldsymbol{C}_{F^{H R}, C R}=\boldsymbol{V}^{0} \otimes \beta^{1} \\
& \boldsymbol{C}_{C R, C R}=\boldsymbol{S}_{1} \text {. }
\end{aligned}
$$

\section{Transient and Stationary Distribution}

The transient and the stationary distribution are calculated in this section.

\section{The Transient Distribution}

Initially, the system is new and the repairperson begins a vacation. For this reason, the initial distribution for the system is $\boldsymbol{\theta}=(\boldsymbol{\alpha} \otimes \boldsymbol{v}, \mathbf{0})$. The transient distribution probability of being in state $i$ at time $v$ is given by the $\mathrm{i}-t h$ element of $\mathbf{p}^{\mathbf{v}}=\boldsymbol{\theta} \mathbf{P}^{\mathbf{v}}$. This vector can be partitioned according to the macro-states as $\boldsymbol{p}_{i}^{v}$ for $i=$ $1, \cdots, 7$

\section{The Stationary Distribution}

The stationary distribution has been worked out by using matrix-algorithmic methods. The stationary distribution $\pi$ is partitioned according to the macro-state space, $\pi=\left\{\pi_{1}, \pi_{2}, \pi_{3}, \pi_{4}, \pi_{5}, \pi_{6}, \pi_{7}\right\}$. This vector verifies the balance matrix equation $\pi \mathbf{P}=\pi$. If the transition probability matrix is expressed as:

$$
\boldsymbol{P}=\left(\begin{array}{ccccccc}
\boldsymbol{C}_{11} & \boldsymbol{C}_{12} & \boldsymbol{C}_{13} & \boldsymbol{C}_{14} & \boldsymbol{C}_{15} & \boldsymbol{C}_{16} & \boldsymbol{C}_{17} \\
\mathbf{0} & \boldsymbol{C}_{22} & \boldsymbol{C}_{23} & \boldsymbol{C}_{24} & \boldsymbol{C}_{25} & \boldsymbol{C}_{26} & \boldsymbol{C}_{27} \\
\mathbf{0} & \mathbf{0} & \boldsymbol{C}_{33} & 0 & \boldsymbol{C}_{35} & 0 & \boldsymbol{C}_{37} \\
\mathbf{0} & \mathbf{0} & \mathbf{0} & \boldsymbol{C}_{44} & \boldsymbol{C}_{45} & \boldsymbol{C}_{46} & \boldsymbol{C}_{47} \\
\boldsymbol{C}_{51} & \mathbf{0} & \mathbf{0} & \mathbf{0} & \boldsymbol{C}_{55} & \mathbf{0} & \mathbf{0} \\
\mathbf{0} & \mathbf{0} & \mathbf{0} & \mathbf{0} & \mathbf{0} & \boldsymbol{C}_{66} & \boldsymbol{C}_{67} \\
\boldsymbol{C}_{71} & \mathbf{0} & \mathbf{0} & \mathbf{0} & \mathbf{0} & \mathbf{0} & \boldsymbol{C}_{77}
\end{array}\right)
$$

The equations can be expressed jointly with the normalization equation by matrix blocks as:

$$
\begin{aligned}
& \pi_{1}=\pi_{1} C_{11}+\pi_{5} C_{51}+\pi_{7} C_{71}, \\
& \pi_{2}=\pi_{1} C_{12}+\pi_{2} C_{22}, \\
& \pi_{3}=\pi_{1} C_{13}+\pi_{2} C_{23}+\pi_{3} C_{33}, \\
& \pi_{4}=\pi_{1} C_{14}+\pi_{2} C_{24}+\pi_{7} C_{44}, \\
& \pi_{5}=\pi_{1} C_{15}+\pi_{2} C_{25}+\pi_{3} C_{35}+\pi_{4} C_{45}+\pi_{5} C_{55}, \\
& \pi_{6}=\pi_{1} C_{16}+\pi_{2} C_{26}+\pi_{4} C_{46}+\pi_{6} C_{66}, \\
& \pi_{7}=\pi_{1} C_{17}+\pi_{2} C_{27}+\pi_{4} C_{47}+\pi_{6} C_{67}+\pi_{7} C_{77},
\end{aligned}
$$

and $\sum_{i=1}^{7} \pi_{i} e=1$.

The solution in a matrix form has been worked out by using matrix-analytics methods and it is given by:

$$
\pi_{i}=\pi_{1} \boldsymbol{R}_{i} ; i=1, \ldots, 7,
$$

where: 


$$
\begin{aligned}
\boldsymbol{G}_{i} & =\left(I-\boldsymbol{C}_{i i}\right)^{-1} ; i=2, \ldots, 7, \\
\boldsymbol{H}_{i} & =\boldsymbol{C}_{1 i}+\boldsymbol{C}_{12} \boldsymbol{G}_{2} \boldsymbol{C}_{2 i} ; i=2, \ldots, 7, \\
\boldsymbol{R}_{2} & =\boldsymbol{C}_{12} \boldsymbol{G}_{2}, \\
\boldsymbol{R}_{i} & =\boldsymbol{H}_{i} \boldsymbol{G}_{i} ; i=3,4, \\
\boldsymbol{R}_{5} & =\left(\boldsymbol{H}_{5}+\boldsymbol{H}_{3} \boldsymbol{G}_{3} \boldsymbol{C}_{35}+\boldsymbol{H}_{4} \boldsymbol{G}_{4} \boldsymbol{G}_{45}\right) \boldsymbol{G}_{5}, \\
\boldsymbol{R}_{6} & =\left(\boldsymbol{H}_{6}+\boldsymbol{H}_{4} \boldsymbol{G}_{4} \boldsymbol{C}_{46}\right) \boldsymbol{G}_{6} \\
\boldsymbol{R}_{7} & =\left(\boldsymbol{H}_{7}+\boldsymbol{H}_{3} \boldsymbol{G}_{3} \boldsymbol{C}_{37}+\boldsymbol{H}_{4} \boldsymbol{G}_{4} \boldsymbol{C}_{47}\right. \\
& \left.+\boldsymbol{H}_{6} \boldsymbol{G}_{6} \boldsymbol{C}_{67}+\boldsymbol{H}_{4} \boldsymbol{G}_{4} \boldsymbol{C}_{46} \boldsymbol{G}_{6} \boldsymbol{C}_{67}\right) \boldsymbol{G}_{7}
\end{aligned}
$$

The vector $\pi_{1}$ is given by:

$$
\pi_{1}=(\mathbf{1}, \mathbf{0})\left(\boldsymbol{e}+\sum_{i=2}^{7} \boldsymbol{R}_{2} \boldsymbol{e} \mid\left(\mathbf{C}_{11}+\mathbf{R}_{5} \mathbf{C}_{51}+\mathbf{R}_{7} \mathbf{C}_{71}-\mathbf{I}\right)^{*}\right)^{-1},
$$

where, $*$ denotes the removing of the first column of the matrix.

\section{Measures}

Several interesting reliability measures are calculated in this section.

\section{Availability}

It is the probability that the system is operational. It is given by:

$$
A(v)=\sum_{i=1}^{4} \boldsymbol{p}_{i}^{v} \boldsymbol{e}
$$

In the steady-state case, the availability is the proportional time that the system is operational, $A=\sum_{i=1}^{4} \pi_{i} e$.

\section{Reliability}

The time up to the first time that the system is not operational is phase-type distributed with representation $\left((\alpha \otimes v, 0), \mathbf{P}^{\prime}\right)$ where:

$$
\boldsymbol{P}^{\prime}=\left(\begin{array}{cccc}
\boldsymbol{C}_{O_{1}, O_{1}} & \boldsymbol{C}_{O_{1}, O_{2}^{W R}} & \boldsymbol{C}_{O_{1}, O_{2}^{R}} & \boldsymbol{C}_{O_{1}, O_{3}^{W R}} \\
\mathbf{0} & \boldsymbol{C}_{O_{2}^{W R}, O_{2}^{W R}} & \boldsymbol{C}_{O_{1}^{W R}, O_{2}^{R}} & \boldsymbol{C}_{O_{2}^{W R}, O_{3}^{W R}} \\
\mathbf{0} & \mathbf{0} & \boldsymbol{C}_{O_{2}^{R}, O_{2}^{R}} & \mathbf{0} \\
\mathbf{0} & \mathbf{0} & \mathbf{0} & \boldsymbol{C}_{O_{3}^{W R}, O_{3}^{W R R}}
\end{array}\right)
$$

Therefore, the probability of being operational the system at time $v$ is given by $(\boldsymbol{\alpha} \otimes v, \mathbf{0})\left(\mathbf{I}-\boldsymbol{P}^{\prime}\right)^{-1} \mathbf{P}^{\mathrm{v}} \mathbf{e}$. The mean time that the system is operational up to first failure is $(\boldsymbol{\alpha} \otimes \mathbf{v}, \mathbf{0})\left(\mathbf{I}-\mathbf{P}^{\prime}\right)^{-1} \mathbf{e}$.

\section{Conditional Probability of Failure}

The conditional probability of failure is the probability of failing at a certain time $\mathbf{v}$. It is given by:

$$
\begin{aligned}
F(v) & =\boldsymbol{p}_{1}^{v-1}\left(\boldsymbol{T}_{1}^{0} \otimes \boldsymbol{e}_{w}\right)+\boldsymbol{p}_{2}^{v-1}\left(\boldsymbol{T}_{2}^{0} \otimes \boldsymbol{e}_{w}\right) \\
& +\boldsymbol{p}_{3}^{v-1} \boldsymbol{T}_{2}^{0}+\boldsymbol{p}_{3}^{v-1}\left(\boldsymbol{T}_{3}^{0} \otimes \boldsymbol{e}_{w}\right) .
\end{aligned}
$$

This probability in steady-state is given by:

$$
\begin{aligned}
F & =\pi_{1}\left(\boldsymbol{T}_{1}^{0} \otimes \boldsymbol{e}_{w}\right)+\pi_{2}\left(\boldsymbol{T}_{2}^{0} \otimes \boldsymbol{e}_{w}\right)+\pi_{3} \boldsymbol{T}_{2}^{0} \\
& +\pi_{3}\left(\boldsymbol{T}_{3}^{0} \otimes \boldsymbol{e}_{w}\right) .
\end{aligned}
$$

Mean operational time, mean non-operational time, mean time that the repairperson is on-line and in vacations.

The mean time that the system is in state $i$ is given by the i-th element of the matrix

$$
M(v)=\theta \sum_{n=0}^{v} \boldsymbol{P}^{n} \boldsymbol{e}
$$

From this result, the following measures are defined in transient and stationary regime.

\section{Mean Operational Time}

Mean time that the system is operational up to time $v$. It is given by:

$$
M_{O_{p}}(v)=\sum_{n=0}^{v}\left(\boldsymbol{p}_{1}^{n} \boldsymbol{e}_{n_{1} w}+\boldsymbol{p}_{2}^{n} \boldsymbol{e}_{n_{2} w}+\boldsymbol{p}_{3}^{n} \boldsymbol{e}_{n_{2}}+\boldsymbol{p}_{4}^{n} \boldsymbol{e}_{n_{3} w}\right)
$$

The steady-state case is the operational time rate $M_{O_{p}}=\pi_{1} \boldsymbol{e}_{n_{1} w}+\pi_{2} \boldsymbol{e}_{n_{2} w}+\pi_{3} \boldsymbol{e}_{n_{2}}+\pi_{4} \boldsymbol{e}_{n_{3} w}$.

\section{Mean Non-Operational Time}

Mean time that the system is non-operational up to time $v$. It is given by:

$$
M_{N O p}(v)=\sum_{n=0}^{v}\left(\boldsymbol{p}_{5}^{n} \boldsymbol{e}_{z 2}+\boldsymbol{p}_{6}^{n} \boldsymbol{e}_{w}+\boldsymbol{p}_{7}^{n} \boldsymbol{e}_{z 1}\right) .
$$

The long-run case is the non-operational time rate $M_{N O p}=\pi_{5} \boldsymbol{e}_{z_{2}}+\pi_{6} \boldsymbol{e}_{w}+\pi_{7} \boldsymbol{e}_{z_{1}}$.

\section{Mean Time that the Repairperson is on-Line}

Mean time that the repairperson is not on vacations up to time $v$. It is given by:

$$
M_{\text {On-line }}(v)=\sum_{n=0}^{v}\left(\boldsymbol{p}_{3}^{n} \boldsymbol{e}_{n_{2}}+\boldsymbol{p}_{5}^{n} \boldsymbol{e}_{w}+\boldsymbol{p}_{7}^{n} \boldsymbol{e}_{z_{1}}\right)
$$

The rate of time that the repairperson is not on vacations is $M_{\text {On-line }}=\pi_{3} \boldsymbol{e}_{n_{2}}+\pi_{5} \boldsymbol{e}_{w}+\pi_{7} \boldsymbol{e}_{z_{1}}$.

\section{Mean Time that the Repairperson is on Vacations}

Mean time that the repairperson is on vacations up to time $v$. It is given by: 


$$
\begin{gathered}
M_{\text {vacation }}(v)=\sum_{n=0}^{v}\left(\boldsymbol{p}_{1}^{n} \boldsymbol{e}_{n_{1} w}+\boldsymbol{p}_{2}^{n} \boldsymbol{e}_{n_{2} w}+\boldsymbol{p}_{4}^{n} \boldsymbol{e}_{n_{3} w}+\boldsymbol{p}_{6}^{n} \boldsymbol{e}_{w}\right) . \\
\text { The proportional } \quad \text { vacations } \quad \text { time is } \\
M_{\text {vacation }}=\pi_{l} \mathbf{e}_{n_{l} w}+\pi_{2} \mathbf{e}_{n_{2} w}+\pi_{4} \mathbf{e}_{n_{3} w}+\pi_{6} \mathbf{e}_{w} .
\end{gathered}
$$

\section{Costs and Rewards}

The system described in Section 1 is subject to different costs and rewards according to whether it is working or not.

Each time that the system is operational, a reward equal to $B$ is achieved. The system works in macro estate 1,2 or 3 and a cost determined by the respective state is produced. These costs are given by the column vectors $c_{1}, c_{2}$ and $c_{3}$ respectively.

Each time that the system is not operational, a loss equal to $A$ is produced. The repairperson produces a cost depending on the work being performed. If the repairperson is on-line but idle, a cost equal to $r_{S}$ per unit of time is produced. If the repairperson is working on preventive maintenance, a cost depending on the phase of preventive maintenance is given by the column vector $r_{P M}$. If the repairperson is engaged in corrective repair, the column vector cost, depending on the repair phase, is given by $\boldsymbol{r}_{C R}$.

The net rewards according to the macro-states are given by the expressions:

$$
\begin{aligned}
\boldsymbol{n} \boldsymbol{r}_{O_{1}}= & \boldsymbol{B} \boldsymbol{e}_{n_{1} w}-\boldsymbol{c}_{1} \boldsymbol{e}_{w} ; \boldsymbol{n} \boldsymbol{r}_{O_{2}^{W R}}=\boldsymbol{B} \boldsymbol{e}_{n_{2} w}-\boldsymbol{c}_{2} \boldsymbol{e}_{w} \\
\boldsymbol{n} \boldsymbol{r}_{O_{2}^{R}}= & \left(\boldsymbol{B}-r_{s}\right)-\boldsymbol{c}_{2} ; \boldsymbol{n} \boldsymbol{r}_{O_{3}^{W R}}=\boldsymbol{B} \boldsymbol{e}_{n_{3} w}-\boldsymbol{c}_{3} \boldsymbol{e}_{w} \\
\boldsymbol{n} \boldsymbol{r}_{P M}= & -\boldsymbol{A} \boldsymbol{e}_{z 2}-\boldsymbol{r}_{P M} ; \boldsymbol{n} \boldsymbol{r}_{F^{W R}}=-\boldsymbol{A} \boldsymbol{e}_{w} \\
& \boldsymbol{n} \boldsymbol{r}_{C R}=-\boldsymbol{A} \boldsymbol{e}_{z_{1}}-\boldsymbol{r}_{C R} .
\end{aligned}
$$

Therefore, the net reward vector by considering the phases of the system is given by:

$$
\boldsymbol{n} \boldsymbol{r}=\left(\begin{array}{c}
\boldsymbol{n} \boldsymbol{r}_{O_{1}} \\
\boldsymbol{n} \boldsymbol{r}_{O_{2}^{W R}} \\
\boldsymbol{n} \boldsymbol{r}_{O_{2}^{R}} \\
\boldsymbol{n} \boldsymbol{r}_{O_{3}^{W R}} \\
\boldsymbol{n} \boldsymbol{r}_{P M} \\
\boldsymbol{n} \boldsymbol{r}_{F^{W R}} \\
\boldsymbol{n} \boldsymbol{r}_{C R}
\end{array}\right)
$$

The mean net reward function per unit of time up to time $v$ is given by:

$$
\operatorname{MNR}(v)=\frac{1}{v+1} \sum_{n=0} \boldsymbol{p}^{v} \boldsymbol{n} \boldsymbol{r}
$$

This value in the stationary case is:

$M N R=\pi \cdot \mathbf{n r}$.

\section{Numerical Example: An Optimization Problem}

A system as described in this paper is assumed. This general system is composed of multiple internal stages and they are partitioned into minor, moderate and major depending on the damage. There are 11 states which are partitioned as follows: 1-3, the system has undergone minor damage; $4-8$, the system has undergone moderate damage (if it is observed, the repairperson remains in the workspace); 9-11, the system has undergone major damage (if it is observed, the repairperson sends it to preventive maintenance). The repair facility is composed of one repairperson. This repairperson can take vacations (the vacation time is random in this general case). When the system is in moderate state or it fails and is observed by the repairperson (he is in the workplace), the preventive maintenance or corrective repair begins respectively. The operational, preventive maintenance and corrective repair time are phase type distributed with representation given in Table 1.

Rewards and cost are introduced in the problem. A profit per unit of time equal to $B=50$ occurs whereas the system is operational (this will be the lost when the system is not operational, $A=50$ ). While the system is operational a cost is produced depending on the operational phase in the following way $c_{1}=(5,6,7)^{\prime}, c_{2}$ $=(8,9,10,11,12)^{\prime}$ ' and $\mathbf{c}_{3}=(30,31,40)^{\prime}$. Each time unit that the repairperson is the workspace waiting for major damage or failure to occur, a cost equal to $r_{S}=80$ is produced. This amount increases when the repairperson is working. If the repairperson is engaged in preventive maintenance, the cost increases by 0,5 and 10 monetary units depending on the preventive maintenance state, respectively. For corrective repair, the amount increases by 20,30 and 40 monetary units, respectively. In the following, we examine how the repairperson's vacation time should be distributed to optimise net rewards. To do so, two situations are considered. First, the situation in which vacation time is distributed geometrically, whereby the probability of the system failing at any time is equal to $1-p$. And second, the situation in which the distribution of the vacation time is phase type (discrete gamma distribution) with representation:

$$
\left.(1,0),\left(\begin{array}{cc}
p_{1} & 1-p_{1} \\
0 & p_{2}
\end{array}\right)\right) .
$$


Table 1: Operational, corrective repair and preventive maintenance time distributions

\begin{tabular}{|c|c|c|c|c|c|c|c|c|c|c|c|}
\hline \multicolumn{12}{|c|}{$\begin{array}{l}\text { Operational Time } \\
\alpha=(\mathbf{1 , 0 )}\end{array}$} \\
\hline \multirow{11}{*}{$\boldsymbol{T}=$} & 0.7 & 0.2 & 0.08 & 0.003 & 0.002 & 0.001 & 0.005 & 0.001 & 0.001 & 0.002 & 0.002 \\
\hline & 0.35 & 0.25 & 0.35 & 0.01 & 0.003 & 0.015 & 0.003 & 0.002 & 0.01 & 0.003 & 0.002 \\
\hline & 0.5 & 0.3 & 0.15 & 0.003 & 0 & 0.005 & 0.001 & 0.02 & 0.014 & 0.002 & 0.003 \\
\hline & 0 & 0 & 0 & 0.5 & 0.1 & 0.1 & 0.1 & 0.15 & 0.025 & 0.01 & 0.01 \\
\hline & 0 & 0 & 0 & 0.6 & 0.25 & 0.05 & 0.02 & 0.03 & 0.03 & 0.0015 & 0.002 \\
\hline & 0 & 0 & 0 & 0.02 & 0.3 & 0.1 & 0.2 & 0.155 & 0.01 & 0.02 & 0.01 \\
\hline & 0 & 0 & 0 & 0.3 & 0.2 & 0.253 & 0.15 & 0.05 & 0.02 & 0.01 & 0.015 \\
\hline & 0 & 0 & 0 & 0.1 & 0.1 & 0.2 & 0.355 & 0.2 & 0.01 & 0.02 & 0.01 \\
\hline & 0 & 0 & 0 & 0 & 0 & 0 & 0 & 0 & 0.15 & 0.3 & 0.4 \\
\hline & 0 & 0 & 0 & 0 & 0 & 0 & 0 & 0 & 0 & 0.4 & 0.5 \\
\hline & 0 & 0 & 0 & 0 & 0 & 0 & 0 & 0 & 0.1 & 0.2 & 0.5 \\
\hline
\end{tabular}

Mean Time up to Failure:50:1014u.t.

Corrective Repair Time

$\beta^{1}=(1,0,0)$

$\boldsymbol{S}_{1}=\left(\begin{array}{lll}0.2 & 0.4 & 0.3 \\ 0.2 & 0.2 & 0.5 \\ 0.3 & 0.2 & 0.3\end{array}\right)$

Mean Corrective Repair Time:7:3810u.t.

Preventive maintenance time

$\beta^{2}=(1,0,0)$

$\boldsymbol{S}_{2}=\left(\begin{array}{lll}0.2 & 0.3 & 0.1 \\ 0.1 & 0.1 & 0.4 \\ 0.2 & 0.2 & 0.2\end{array}\right)$

Mean Preventive Maintenance Time:2:5u.t.

Table 2: Main stationary regime measures for case I

\begin{tabular}{llll}
\hline Rate Operational Time & Rate non-operational time & $\begin{array}{l}\text { Rate time that } \\
\text { the repairperson is on-line }\end{array}$ & $\begin{array}{l}\text { Rate time that the } \\
\text { repairperson is on vacations }\end{array}$ \\
\hline $\mathrm{M}_{O p}=0.9287$ & $M_{N O p}=0.0713$ & $M_{\text {On-line }}=0.3236$ & $M_{\text {vacation }}=0.6764$
\end{tabular}

Table 3: Main stationary regime measures for case II

\begin{tabular}{llll}
\hline Rate Operational Time & Rate non-operational time & $\begin{array}{l}\text { Rate time that the } \\
\text { repairperson is on-line }\end{array}$ & $\begin{array}{l}\text { Ratetime that the } \\
\text { repairperson is on vacations }\end{array}$ \\
\hline$M_{O p}=0.9280$ & $M_{N O p}=0.0720$ & $M_{\text {On-line }}=0.3217$ & $M_{\text {vacation }}=0.6783$
\end{tabular}

\section{Case I: The Geometric Case for the Vacations Time}

We assume that the repairperson keeps on vacations with probability equal to $p$ at any time. The optimum operational time occurs when the repairperson has not vacations whether costs are not considered, obviously. For this case, the rate operational time is 0:9338 and the system provides a profit equal to 9:7102 monetary units per unit of time. But, the system has to be optimized by considering costs and rewards. For this, the net reward function in the stationary regime, given in Section 5, is considered. Figure 1 shows this function.

The optimum value is reached for $p=0: 4525$ and it is equal 9:7613 monetary units per unit of time. For this case the main steady-state measures calculated in Section 4 are given in Table 2.

\section{Case II: The Discrete Gamma Case for the Vacations Time}

We assume that the repairperson keeps on vacations according to a probability distribution phase type with representation (3). The optimum operational time occurs when $\mathrm{p} 1=0$ and $\mathrm{p} 2=0$. For this case the rate operational time is 0:6726 and the system provides a profit equal to 9:7789 monetary units per unit of time. But, again the system has to be optimized by considering costs and rewards. For this, the net reward function in the stationary regime, given in (2), is considered. Figure 2 shows this function. 


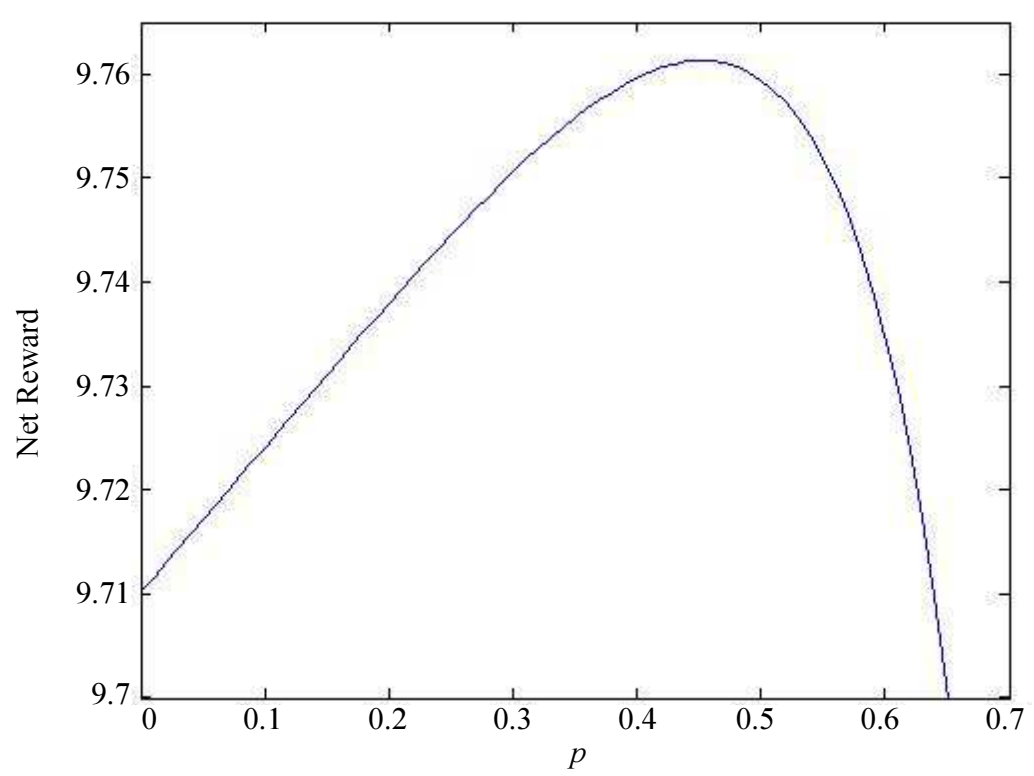

Fig. 1: Net reward versus $p$

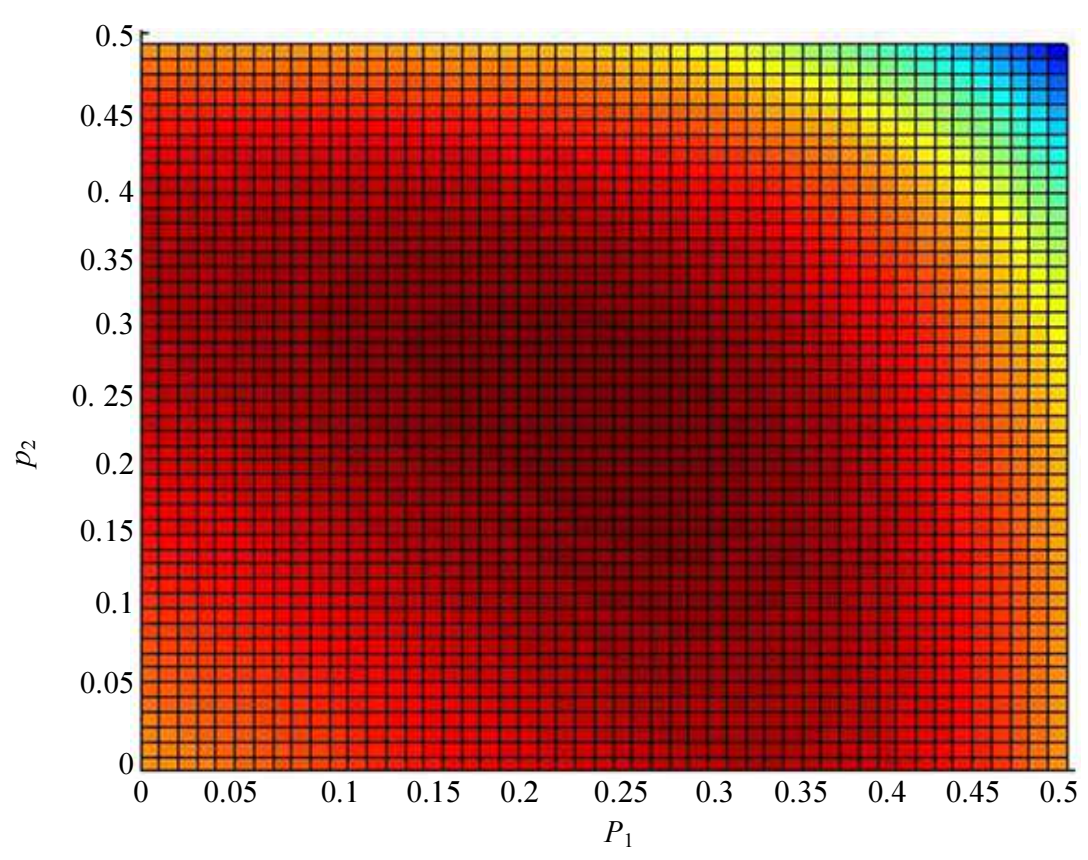

Fig. 2: Net reward versus $p_{1}$ and $p_{2}$

The optimum value is reached for $\mathrm{p} 1=0: 2386$ and $\mathrm{p} 2$ $=0: 2386$ and the net reward is equal 9:7924 monetary units per unit of time. For this case the main steady-state measures calculated in Section 4 are given in Table 3.

\section{Conclusion}

In this paper, a multi-state system subject to repairable failure and which receives preventive maintenance is modelled. This multi-state system can pass through any of three damage levels (composed of multiple states): minor, moderate and major. The repair facility is composed of a single repairperson. To optimise the model from an economic standpoint, the repairperson begins randomly-timed vacations after each repair. When the repairperson returns, four possible situations can occur; the system is at the first damage level (the repairperson begins a new randomly-timed vacation), the system is at the second damage level (the repairperson remains in the workspace waiting for a fatal transition to occur), the system is at the third damage level (the repairperson begins preventive 
maintenance) and finally, the system is broken (the repairperson begins corrective repair).

The system is modelled in an algorithmic and computational form and the main results can be treated computationally. All times embedded in the system are phase-type distributed. This fact enables to express the transient and stationary distributions and measures in a well structured way. Given that any discrete time distribution with finite support is a phase type distribution, the modelling can be considered general for this case.

Cost and rewards are included in the model and several associated measures are worked out. One interesting measure, the net reward function, is built and it is considered to optimize a system according to the vacation time distribution.

\section{Acknowledgements}

This paper is partially supported by the Junta de Andalucía, Spain, under the grant FQM-307 and by the Ministerio de Economía y Competitividad, España, under Grant MT M2017 - 88708 - P and by the European Regional Development Fund (ERDF).

\section{Ethics}

The author declares that there is no conflict of interests regarding the publication of this article.

\section{References}

Arulmozhi, G. and R. Nadarajan, 1994. Markovian parallel system with the repairmen vacation. Microelectronics Reliability, 34: 947-950.

Lisnianski, A. and I. Frenkel, 2012. Recent advances in system reliability. Signatures, Multi-state Systems and Statistical Inference. Springer-Verlag, London.

Lisnianski, A., I. Frenkel and A. Karagrigoriou, 2018. Recent advances in multi-state systems reliability. Theory and Applications. Springer International Publishing AG.

Murchland, J., 1975. Fundamental concepts and relations for reliability analysis of multi state systems. In Reliability and Fault Tree Analysis, Theoretical and Applied Aspects of System Reliability and Safety Assessment. SLAM, Philadelphia.
Nakagawa, T., 1977. Optimum preventive maintenance policies for repairable systems. IEEE Trans. Reliability, R-26: 168-173.

Nakagawa, T., 2005. Maintenance theory of reliability. Springer Series in Reliability Engineering.

Neuts, M., 1975. Probability distributions of phase type. In: Liber Amicorum, Florin, E.H. (Eds.), Belgium: Department of Mathematics, University of Louvain, pp: 173-206.

Neuts, M., 1981. Matrix geometric solutions in stochastic models. An algorithmic approach. Dover Publications.

Ruiz-Castro, J. and G. Fernández-Villodre, 2012. A complex discrete warm standby system with loss of units. Eur. J. Operational Res., 218: 456-469.

Ruiz-Castro, J. and M. Dawabsha, 2018. Modelling a multi-state k-out-of-n: G system with loss of units. Reliability Engineering. Theory and Applications, Taylor and Francis/CRC Press, pp: 43-60.

Ruiz-Castro, J., 2016a. Complex multi-state systems modelled through marked Markovian arrival processes. Eur. J. Operational Res., 252: 852-865.

Ruiz-Castro, J., 2016b. Markov counting and reward processes for analysing the performance of a complex system subject to random inspections. Reliability Engineering System Safety, 145: 155-168.

Ruiz-Castro, J., 2018. A d-mmap to model a complex multi-state system with loss of units. Recent Advances in Multi-State Systems Reliability, Springer, pp: 39-59.

Takagi, H., 1991. Queueing analysis: A foundation of performance evaluation vacation and priority systems. North-Holland: Amsterdam.

Tian, N. and Z. Zhang, 2006. Vacation Queueing Models-Theory and Applications. Berlin: Springer.

Zhong, C. and H. Jin, 2014. A novel optimal preventive maintenance policy for a cold standby system based on semi-Markov theory. Eur. J. Operational Res., 232: 405-411. 